\title{
The Connected Past 2017 : archéologie, histoire et complexité. Perspectives présentes et futures de la recherche
}

\author{
Oliver Panichi \\ Université de Teramo (Italie), doctorant de l'École doctorale History of \\ Europe from the Middle Ages to the Contemporary Age
}

Soumis le 13 octobre 2017, mis en ligne le 23 mars 2018

Texte traduit par Silvia Marzagalli

\section{Actualités - News}

"Avantages», "limites», « défis » ont été les mots clés les plus employés dans les communications des 18 intervenant.e.s qui se sont retrouvé.e.s à Bournemouth (Royaume-Uni) pour la rencontre The Connected Past $2017^{1}$.

L'application des techniques d'analyse de réseaux à la recherche historique et surtout archéologique constitue désormais un acquis dans le panorama scientifique international. L'innovation se poursuit à un rythme très soutenu grâce à la mise à disposition d'un nombre de plus en plus grand de technologies informatiques permettant de collecter les données, de construire des modèles et de formuler des hypothèses relatives aux actions individuelles et collectives passées. Des groupes de travail pluridisciplinaires sont à même de produire des études de plus en plus solides du point de vue mathématique et statistique. La simulation informatique de mondes possibles, de systèmes virtuels peuplés par des sujets mis en scène par des algorithmes laisse entrevoir de grandes possibilités d'élargir nos connaissances sur des réalités très

1 The Connected Past 2017: The Future of Past Networks?, 24-25 août 2017, Bournemouth University, Bournemouth (UK). Organisateurs et organisatrices : Fiona Coward, Anna Collar et Tom Brughmans.

ARCS 
éloignées dans le temps et/ou qui ont laissé très peu de traces historiques et archéologiques.

C'est précisément à cause du taux élevé d'innovation que l'on constate dans ces études que la présentation des recherches et des résultats ne peut pas faire l'économie d'une réflexion sur la méthodologie, et plus particulièrement d'une évaluation constante des potentialités et des limites relatives à chaque technique informatique utilisée.

On invite par ailleurs depuis des années, dans la communication scientifique dans ce domaine, à considérer les techniques et les méthodes de travail comme des moyens, et pas comme des fins en soi. Ces invitations continuent d'être extrêmement nécessaires, comme l'a précisé l'archéologue Tom Brughmans, l'un des organisateurs de la rencontre: "Ce qu'il faut avant toute chose, c'est une connaissance solide des méthodes pour appliquer l'analyse de réseaux et les modèles multi-agents à nos travaux, les choisissant et les utilisant lorsqu'ils sont vraiment nécessaires, et pas, comme cela est encore souvent le cas, pour démontrer qu'on sait les utiliser. Je crois qu'il convient de se poser à chaque fois la question : à quoi cela sert pour de vrai ? En quoi cela peut enrichir notre recherche? »

\section{Archéologie et informatique : l'apport de l'analyse de réseaux}

Au cours de The Connected Past 2017, les présentations situées dans le champ de l'archéologie ont été plus nombreuses que celles situées en histoire. Le recours à des techniques informatiques pour l'étude archéologique des peuplements humains a été l'objet principal de la conférence inaugurale d'Eleftheria Paliou ${ }^{2}$, qui a présenté les innovations les plus récentes dans ce domaine. Elle a rappelé que le fait de penser les individus et les espaces en termes de réseaux, nœuds et relations constitue une approche présente déjà depuis un certain temps en sciences humaines et sociales : par contre, ce qui a émergé avec force au cours des dernières années, c'est le développement de techniques de travail adaptées aux exigences spécifiques de l'archéologie. Parmi les champs d'application les plus prometteurs, il y a la reconstitution des réseaux de transport, l'identification des raisons pour lesquelles un site

2 Eleftheria Paliou, "Network science and the archaeology of human settlements". 
urbain s'est développé dans un endroit plutôt que dans un autre, l'analyse du comportement des individus à travers les espaces naturels et artificiels, les recherches sur les champs visuels des individus dans un environnement donné. Ce dernier aspect a été développé plus spécifiquement par Tom Brughmans qui, dans sa présentation avec Ulrik Brandes, a discuté la possibilité d'appliquer les techniques de l'analyse des réseaux à l'archéologie du paysage ${ }^{3}$. Le point clé ici est représenté par le rapport entre les champs de vision et les comportements humains : de quelle manière un individu est-il influencé par ce qu'il peut voir? quelles sont les actions les plus probables quand les conditions de visibilité du milieu où vit le ou les sujets dont on étudie le comportement se modifient? Il s'agit de questions que l'archéologue peut se poser par rapport aux espaces étudiés. D'après Brughmans, l'apport de la théorie de réseaux consiste en sa possibilité de créer des modèles théoriques et des simulations relatives aux comportements des sujets humains dans le milieu étudié. Grâce à ces représentations virtuelles, il est ensuite possible de vérifier les hypothèses formulées par l'observation empirique de ces mêmes espaces aujourd'hui.

C'est dans ce contexte que l'archéologie, une fois assimilées dans sa méthode de travail les ressources de la science des réseaux (Network science), peut avoir recours aussi aux techniques des modèles multiagents (Agent-Based Modeling, ABM). Il s'agit d'une simulation informatique du passé, même lorsque celui-ci est très éloigné dans le temps, moyennant la création de systèmes virtuels que les chercheurs et chercheuses peuplent d'individus dont les règles de comportement et d'interaction sont créées et modifiées par le recours à des outils mathématiques. Dans son intervention, Lennart Linde a défini les techniques $A B M$ comme des «laboratoires informatiques" («computational laboratories») dans lesquels les archéologues peuvent générer des réseaux virtuels, où ils font agir les dynamiques de fonctionnement qu'ils estiment être à l'œuvre dans les réseaux archéologiques étudiés ${ }^{4}$.

Jusqu'à quelle époque passée peut-on employer cette technique? Dans sa communication, Iza Romanowska a illustré l'emploi des

3 Tom Brughmans, Ulrik Brandes, "New methods for visibility networks in landscape archaeology".

4 Lennart Linde, "Networks, Agents and Interactions: How to use Agent Based Models to Investigate Archaeological Network Formation".

ARCS 
techniques des modèles multi-agents à l'aube du genre humain, proposant une simulation des comportements d'un groupe d'hominidés, différenciés en fonction de leur plus ou moins grande capacité d'adaptation à leur environnement, pour formuler des hypothèses sur les dynamiques de croissance et les dynamiques migratoires d'une population $^{5}$.

\section{La recherche archéologique à l'épreuve de la pénurie/abondance des données}

La réflexion sur les limites et les contraintes imposées par la nature des données étudiées a été un élément commun à la majeure partie des interventions. Le caractère incomplet et fragmentaire des données constitue une situation très fréquente pour les archéologues et pourtant, des recherches présentées dans ce secteur avaient comme principal défi la surabondance d'informations à interpréter. Le groupe de recherche constitué par Aurélia Feugnet, Clara Filet et le mathématicien Fabrice Rossi a ainsi étudié un corpus de 57735 artefacts retrouvés dans les territoires peuplés par les Celtes entre le IIIe et le Ier millénaire av. J.-C6. S'agissant de produits manufacturés provenant sûrement de la Méditerranée, leur étude peut fournir beaucoup d'informations sur les dynamiques commerciales entre l'Europe du Nord et du Sud à cette époque. Nous sommes toutefois devant un cas classique où l'abondance d'information pose potentiellement problème. Dans cette étude, le recours à la formalisation mathématique a permis de rendre davantage lisible la distribution des artefacts entre les différents sites et d'identifier les coprésences des mêmes types de produits manufacturés dans les mêmes lieux. Par une étude statistique, on a pu évaluer quelles coprésences étaient les plus significatives. La recherche a identifié les spécificités régionales des importations de produits manufacturés en mettant en lumière $\mathrm{d}^{\prime}$ importantes différences entre l'Europe occidentale et l'Europe centrale par rapport aux approvisionnements méditerranéens.

5 Iza Romanowska, "Modelling the evolution of human plasticity: an ABM approach".

6 Clara Filet, Fabrice Rossi, Aurélia Feugnet, "Co-presence Analysis and Significance Scores: when network studies highlight choices of Iron Age elites". 
Un corpus moins large, mais de dimension importante (450 textes), a été traité par Lena Tambs dans son étude de la vie socio-économique du camp militaire de Pathyris, proche de Thèbe, dans l'Egypte ptolémaïque (165-88 av. J.-C.) ${ }^{7}$. Ces témoignages écrits donnent des indications sur la vie quotidienne dans une petite communauté au cours de trois générations. En ayant recours à l'analyse de réseaux sociaux, Tambs a tracé le cadre des relations entre les individus mentionnés par les documents et s'est interrogée sur les problèmes posés par l'attribution des catégories sociales à ceux-ci. La terminologie égyptienne pour indiquer les relations de parentèle et les professions ne sont en effet pas superposables à nos termes contemporains, ce qui pose aux interprètes la nécessité de s'orienter entre les avantages et les limites propres à une approche " émique » et " étique » de la réalité étudiée. Dans cette étude, l'analyse des réseaux croise une réflexion sur notre approche gnoséologique au passé et, plus largement, soulève la vaste question de l'observation scientifique et ses implications en sciences sociales.

\section{L'espace interprété comme un réseau : évolution et mouvements}

Les interventions qui ont directement affronté les limites posées par les lacunes de la documentation archéologique ont en commun l'application de la science des réseaux au thème des espaces et de leur articulation.

En choisissant d'étudier les processions religieuses à Ostie à l'époque romaine, Katherine Crawford traite un fait social d'une époque ancienne rarement documenté par des artefacts archéologiques, en dehors de quelques réalisations architecturales occasionnelles lors des célébrations ${ }^{8}$. Son étude vise à dépasser l'approche adoptée jusqu'à présent, fondée principalement sur les comptes rendus livrés par la littérature classique. Crawford a appliqué des techniques d'analyse de réseaux aux espaces urbains de l'ancienne Ostie (bâtiments publics et habitations privées, lieux de commerce et de production) pour identifier les itinéraires les plus plausibles des processions religieuses et comparer ces hypothèses avec les données issues des vestiges archéologiques. Par

7 Lena Tambs, "Mixing Emic and Etic Perspectives for Studying Small-Scale Communities of the Past: The Case of Pathyris (c. 165-88 BCE)".

8 Katherine Crawford, "Movement within the Cityscape: the potential of applying network methods".

ARCS 
ces simulations, il est possible de récréer le contexte social et architectonique dans lequel ces événements rituels se déroulaient et de tester une série d'hypothèses sur le rapport entre espace urbain et religion à l'époque romaine.

Mark Groenhuijzen a présenté une partie des résultats d'un projet plus vaste appelé Rechercher les limites du limes (Finding the limits of the limes), consacré à l'étude des relations économiques à l'époque romaine entre les légions militaires et les populations locales dans l'actuelle Hollande ${ }^{9}$. Sa recherche s'interroge sur les modalités d'organisation des transports et des approvisionnements pour les légions, afin de comprendre le fonctionnement de la distribution des marchandises et d'identifier les flux les plus probables entre les différents sites. Dans son cas, la faible quantité de vestiges archéologiques a rendu nécessaire le recours à l'informatique pour construire et visualiser des modèles virtuels de ces possibles réseaux de transport. Cette opération a simultanément permis d'émettre une série d'hypothèses sur l'objet d'étude et de discuter des avantages et limites de chaque technique informatique. Par exemple, selon Groenhuijzen, il est permis de douter de l'utilité heuristique d'une technique informatique si celle-ci produit des connexions qui semblent extrêmement irréalistes et qui ne sont pas plausibles à la lumière de connaissances déjà acquises.

Une interrogation transversale à plusieurs communication a été celle du temporal change, à entendre ici comme l'analyse diachronique d'un objet d'étude archéologique ou historique, interprétable par la théorie des réseaux. À ce propos, il convient de préciser le sens très large dans lequel il faut entendre le terme de "réseau ». Plusieurs interventions de cette rencontre ont confirmé que la science des réseaux peut être appliquée avec profit à des objets d'étude qui ne sont pas des réseaux d'individus qui interagissent via des relations sociales (par exemple, de parentèle, économiques, de subordination). Cécile Rivals a ainsi étudié l'évolution urbanistique d'un petit bourg médiéval du sud de la France entre le XIVe et le XIXe siècle en mettant à profit les terriers et les compoix produits sous l'Ancien Régime pour identifier des portions de territoires à des fins fiscales ${ }^{10}$. Ces registres, ancêtres du cadastre

9 Mark Groenhuijzen, Philip Verhagen, "(Finding the Limits of) Network Approaches to Local Transport in the Dutch Part of the Roman Limes".

10 Cécile Rivals, "The modeling of urban spatial dynamics in long time spans: The use of graph theory in Saint-Antonin- Noble-Val (Tarn-et-Garonne, France) 
napoléonien, contiennent des références topologiques mais n'incluent pas de références cartographiques. Est-il possible de les comparer avec les plans cadastraux de l'époque post-napoléonienne? La recherche de Rivals montre que cela est possible une fois que les deux types de documents - registres et plans - ont été conceptualisés comme des réseaux et représentés visuellement comme de graphes. La comparaison des graphes ainsi générés offre des éléments précieux pour reconstruire l'évolution urbaine et rurale de l'espace étudié, ainsi que de la société qui l'a produit au fil des siècles.

\section{Les réseaux sociaux et le changement historique : méthodes et défis}

L'application de la science des réseaux aux réseaux entre individus du passé continue à être un terrain fécond de recherche. Certaines interventions présentées à The Connected Past 2017 ont abordé l'évolution des réseaux sociaux au cours d'une période déterminée, afin de mieux comprendre l'influence des événements extérieurs sur ces réseaux.

Dans son étude historique sur la mission de la Banque mondiale en Colombie en 1949, Elisa Grandi a appliqué l'analyse des réseaux sociaux (ARS) à l'étude de la correspondance entre les membres de la Banque mondiale et les élites économiques et politiques de cet État ${ }^{11}$. Les sources mobilisées couvrent la période 1949-1954 et la méthodologie de l'ARS a permis de décrire leur contenu de manière systématique, notamment la dimension relationnelle entre les individus impliqués (intensité des liens, citations réciproques, identification des individus ayant un rôle de médiation). Cette recherche entend aussi contribuer à une réflexion sur l'approche la plus fonctionnelle, au sein de l'ARS, pour décrire les influences réciproques entre le réseau social étudié et le développement des prêts à la Colombie par la Banque mondiale. D'après Elisa Grandi, l'application du concept des «chaînes relationnelles» proposé par Michel Grossetti permettrait de comprendre de manière plus efficace

from the 14th to the 19th centuries".

11 Elisa Grandi, "Social networks and path dependency in the international economic advising. The World Bank first mission in Colombia and its aftermaths (1949-1954)". 
l'évolution de ce réseau social par rapport aux évolutions du contexte local et global.

La présentation de Grandi et celles d'histoire médiévale montrent une attention pour la dimension des réseaux égocentrés. L'application de l'ARS à l'étude de vastes corpus d'actes officiels, par exemple de testaments, permet d'observer de manière systématique la totalité des interactions dont un individu a été protagoniste, en fonction des traces laissées par celles-ci dans les documents. La communication de Matthew Hammond et Cornell Jackson a présenté quelques aspects du projet People of Medieval Scotland 1093-1286, une base de données prosopographique contenant plus de 6000 profils $^{12}$. Ils ont appliqué des techniques de l'ARS à un corpus de 15000 documents officiels pour identifier les personnalités les plus influentes et la fréquence de leur mention dans les actes en tant que témoins. Cette approche permet aussi de comparer le rôle de plusieurs individus à l'aide des visualisations de leurs réseaux égocentrés. Le projet, bien avancé, a déjà produit un $e$-book disponible gratuitement en ligne avec les graphes et les tableaux produits. La communication présentée au The Connected Past 2017 a été particulièrement utile car elle a proposé quelques pistes méthodologiques pour faire face à la nature parfois incomplète et lacunaire de la base, par exemple pour ce qui concerne l'indication des dates. Hammond est un historien, alors que Jackson est un spécialiste d'ARS. Ces différentes compétences constituent une forte valeur ajoutée pour leur recherche et cela nous semble un exemple à suivre pour tous les projets historiques qui traitent un nombre considérable de documents. Le recours à des compétences informatiques, et en particulier à l'ARS, semble plus développé en archéologie qu'en histoire, du moins à en juger par ce qui a été présenté lors de The Connected Past 2017. Il se peut que la différence d'innovation méthodologique entre les deux disciplines dépende aussi de la moindre ouverture des historien.ne.s à des collaborations avec des spécialistes d'autres disciplines, mathématique, statistique et informatique en particulier.

Un autre projet de base des données prosopographique, tout aussi ambitieux, est celui dont a parlé Christina Williamson dans sa communication consacrée aux jeux panhelléniques organisées dans plusieurs villes grecques dans la période hellénistique et sous

12 Matthew Hammond, Cornell Jackson, "Analysis of Ego-Networks of Witnesses to Medieval Charters: Opportunities and Challenges". 
domination romaine ${ }^{13}$. L'un des objectifs de ce projet appelé Connected contests est d'élaborer et mettre en ligne une base de données prosopographique des athlètes et des organisateurs de ces événements pour lesquels subsistent des traces épigraphiques. Plus largement, ce projet encore en cours se propose d'intégrer une vaste gamme de sources (épigraphiques, numismatiques, littéraires, archéologiques) pour aboutir à une lecture globale de ce phénomène. Les concepts de la science des réseaux semblent bien adaptés pour produire une cartographie suggestive et épistémologiquement utile des flux des athlètes et des ambassadeurs entre les différentes villes/nœuds de ce réseau. "Penser réseaux" ("network thinking") aide, comme l'explicite ouvertement Williamson, en même temps que cela pose des problèmes. Le projet doit prendre en compte des niveaux multiples de réseau, du niveau local et urbain au niveau impérial. Se pose aussi un problème évident en termes de données: comment gérer leur caractère fragmentaire sans compromettre l'efficacité du cadre d'ensemble?

Il s'agit de questions récurrentes, comme nous l'avons signalé. C'est le signe d'un défi intellectuel qui est en train d'évoluer et qui fait face aussi bien à des succès convaincants qu'à des impasses potentiellement préoccupantes. Tout comme un individu dans les phases les plus névralgiques de sa croissance, ce champ d'études se pose beaucoup de questions sur son devenir, sur la direction vers laquelle aller, et en quelle compagnie.

13 Christina Williamson, Onno van Nijf, "Whose network? The complexities of multiscalar festival networks in the Graeco-Roman world". 